\title{
Column Density Measurements of a Prominence Observed by AIA
}

\section{Patrick I. McCauley, Yingna Su, Edward DeLuca, and Adriaan van Ballegooijen}

Harvard-Smithsonian Center for Astrophysics, 60 Garden street, Cambridge, MA 02138, USA

Abstract. We present column density measurements of a polar crown prominence observed on March 9th, 2012 by the Atmospheric Imaging Assembly (AIA) aboard the Solar Dynamics Observatory. The structure was viewed on the east limb by AIA and erupted about 30 hours after the observations shown here. We estimate column density by approximating the obscured background emission to obtain an optical depth. This can then be combined with the absorption cross sections of neutral hydrogen and helium, along with the He:H abundance ratio, to calculate column density. We perform this calculation for the 171, 193, 211, and $335 \AA$ AIA passbands.

Keywords. Sun: prominences - Sun: corona - Sun: coronal mass ejections

\section{Observations and Analysis}

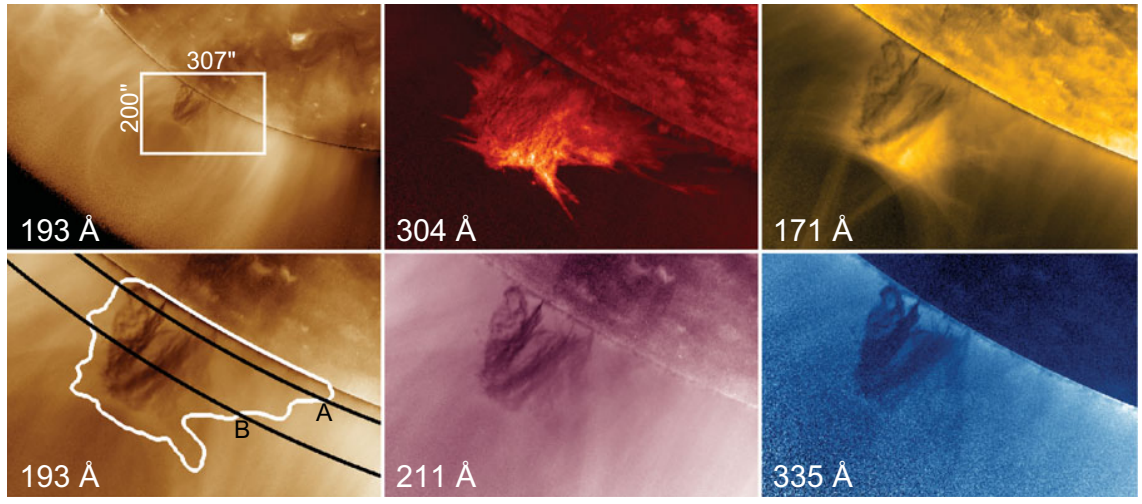

Figure 1. AIA prominence observations taken on 2013/03/09 at 19:35 UT. The white contour on the $193 \AA$ image denotes the $304 \AA$ extent, and the black arcs denote the cuts used in Fig 2 .

To estimate neutral hydrogen column density $\left(N_{\mathrm{HI}}\right)$, we employ a simple technique used in several past studies (e.g. Daw et al. 1995, Kucera et al. 1998, Anzer \& Heinzel $2005)$. Given the observed intensity $\left(I_{o b s}\right)$ across the EUV absorption features in Fig 1, we approximate the obscured background $\left(I_{b}\right)$ and overlying foreground $\left(I_{f}\right)$ using a linear fit to the emission at the intersections of the white contour and concentric slices shown in the lower-left panel. We take $I_{f}$ to be $29 \%$ of this fit based on the deepest absorption. If a larger portion is attributed to $I_{f}$, then $I_{o b s}$ is over-subtracted and Eqn 1.1 is NaN at certain positions. This leads to the optical depth $(\tau)$, which is combined with the ratio of He:H ( $r=15 \%$; Del Zanna et al. 2004) and their absorption cross sections $\left(\sigma_{\mathrm{HI}} \& \sigma_{\mathrm{He} \mathrm{I}}\right.$; Anzer \& Heinzel 2005, West \& Marr 1976) to yield $N_{\mathrm{HI}}$ along the line-of-sight.

$$
I_{o b s}=I_{b} e^{-\tau}+I_{f} \Rightarrow \tau=-\ln \frac{I_{o b s}-I_{f}}{I_{b}}
$$




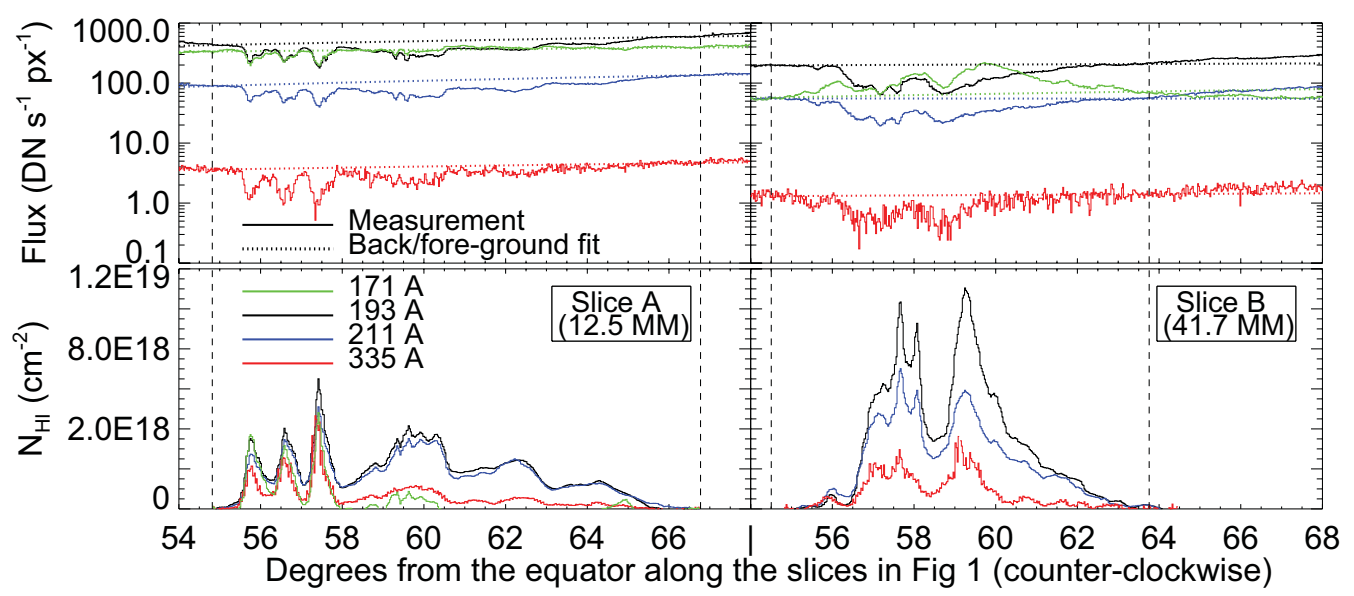

Figure 2. Upper: observed flux. Lower: estimated column densities. Dashed lines denote intersections of white \& black contours in Fig 1, used for linear back/fore-ground fits (dotted).
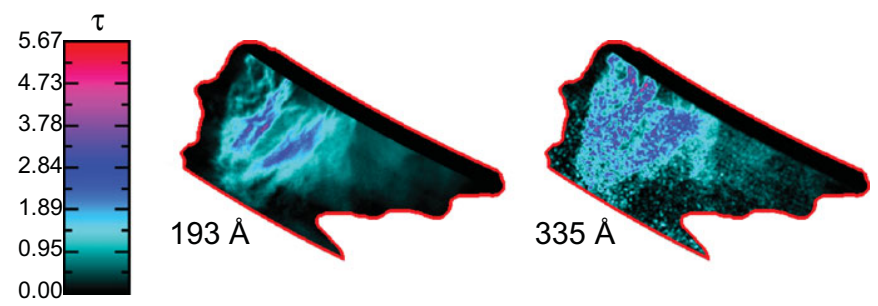

Figure 3. Maps of the optical depth, $\tau$.

$$
\begin{gathered}
\tau=\sigma_{\mathrm{HI}} N_{\mathrm{HI}}+\sigma_{\mathrm{He} \mathrm{I}} N_{\mathrm{He} \mathrm{I}}+\sigma_{\mathrm{He} \mathrm{II}} N_{\mathrm{He} \mathrm{II}} \approx\left(\sigma_{\mathrm{HI}}+r \sigma_{\mathrm{He} \mathrm{I}}\right) N_{\mathrm{HI}} \\
\Rightarrow N_{\mathrm{HI}} \approx \frac{\tau}{\sigma_{\mathrm{HI}}+r \sigma_{\mathrm{He} \mathrm{I}}}
\end{gathered}
$$

We find $N_{\mathrm{HI}}$ to be $\sim 10^{19} \mathrm{~cm}^{-2}$ for the deepest absorption features, which is consistent with similar work (Labrosse et al. 2011). Note that we are sensitive only to the neutral hydrogen, and the ionized fraction likely varies, particularly with height. This, along with a varying filling factor, may explain the discrepancy between the $335 \AA$ channel and the other bands. See $\mathrm{Su}$ et al. (2013) for additional details on this event.

Acknowledgements: This project is supported by NASA grant NNX12AI30G and NASA contract SP02H1701R from LMSAL to SAO.

\section{References}

Anzer, U. \& Heinzel, P. 2005, ApJ, 622, 714

Daw, A., DeLuca, E. E., \& Golub, L. 1995, ApJ, 453, 929

Del Zanna, G., Chiuderi Drago, F., \& Parenti, S. 2004, A\&A, 420, 307

Kucera, T. A., retta V, \& Poland, A. I. 1998, SoPh, 183, 107

Labrosse, N., Schmieder, B., Heinzel, P., \& Watanabe, T. 2011, A\& A, 531, A69

$\mathrm{Su}$, Y., Reeves, K. K., McCauley, P., van Ballegooijen, A., \& DeLuca, E. 2013, Proceedings of IAUS 300: Nature of Prominences and their role in Space Weather

West, J. B. \& Marr, G. V. 1976, Proc. R. Soc. London A, 349, 397 